\title{
SILDENAFIL IS NOT PROTECTIVE TO THE BOWEL FOLLOWING INTESTINAL ISCHEMIA AND REPERFUSION INJURY
}

\author{
Hannah M. Moore, B.S. ${ }^{1}$, Natalie A. Drucker M.D. ${ }^{1,2,3}$, Troy A. Markel M.D. ${ }^{1,2,3}$ \\ ${ }^{1}$ Indiana University School of Medicine, ${ }^{2,3}$ Department of Surgery
}

\section{Background and Hypothesis:}

Acute Mesenteric Ischemia (AMI) occurs when blood supply to the intestine is decreased. This can lead to intestinal ischemia, cellular damage, necrosis, and if corrected, subsequent reperfusion injury. There are currently no medical treatments to help reverse ischemia and reperfusion injury (I/R) and, therefore novel treatments are necessary. Sildenafil, a compound that increases endogenous nitric oxide (NO) by blocking the phosphodiesterase- 5 induced breakdown of cGMP, may function as a potent mesenteric vasodilator. We therefore hypothesized that sildenafil would improve mesenteric perfusion during a mouse model of intestinal I/R.

\section{Experimental Design or Project Methods:}

Adult male C57BI6J mice were anesthetized with isoflurane and a midline laparotomy performed. The base of the superior mesenteric artery was occluded with a non-crushing vascular clamp for 60 minutes. At the end of ischemia, sildenafil $(1 \mathrm{mg} / \mathrm{kg}, 10 \mathrm{mg} / \mathrm{kg}$, or $100 \mathrm{mg} / \mathrm{kg}$ ) or a PBS vehicle control were administered via intraperitoneal injection. Animals were then allowed to recover. Twenty-four hours after ischemia, animals underwent assessment of mesenteric perfusion by Laser-Doppler imaging. Animals were then euthanized. Perfusion was expressed as a percentage of baseline, depicted as mean $+/$ - SEM, and analyzed by ANOVA. $P<0.05$ was significant.

\section{Results:}

There were no significant differences in mesenteric perfusion between the vehicle group or any of the therapeutic groups. (PBS: $53.03 \pm 11.35 \%$; Sildenafil-low: $59.59 \pm 7.55 \%$; Sildenafil-medium: 70.51 \pm 8.49 ; Sildenafil-high: $66.4 \pm 10.2, p=0.61$ ).

\section{Conclusion:}

Sildenafil does not appear to be an effective treatment for improving mesenteric perfusion following intestinal ischemia. Further studies are required to determine the reasons for the ineffectiveness of sildenafil. One possible explanation for these observations could be a lack of PGE5 in the intestinal vascular endothelium. 